\title{
Study of Information Fusion of Distribution Network Automation and Digital Substation Automation
}

\author{
Lei Zhang, Yuzhi Zhou, Bifu Qian, \\ State Grid Wenzhou Electric Power Supply Company, Wenzhou, China
}

\begin{abstract}
Information fusion of distribution network automation and digital substation automation can improve the efficient use of the digital substation's distribution network data and strengthen the distribution network. The scheme adds a distribution network area controller for information interaction and integrally models distribution network and digital substation equipment based on the IEC 61850 protocol. This paper details the information fusion scheme of regional distribution network and substation automation system as well as the main function of the controller. The case study demonstrates that the fusion strategy can make the information collection more direct and the fault elimination more intelligent when dealing with the defects and faults of the distribution network.
\end{abstract}

\section{Introduction}

Digital substation technology is the development trend of substation automation, and the integration of distribution network automation systems and digital substation automation systems is also the future trend. According to the construction plan of the digital substation, IEC61850 modelling is required for distribution network equipment to fully digitize the distribution network and completely integrate the distribution network into the digital substation automation system. [1-3] However, IEC61850 mode of distribution network equipment, together with the relevant standards, is not mature currently. What's more, the construction cost of digital transformation of distribution network equipment is too high. Under current conditions, it is not suitable to fully implement the distribution network digitization in terms of economy and equipment operation status.

Based on the IEC61850 protocol, this paper models the regional distribution network and connects the model to the targeted distribution network area to efficiently control the local distribution network. The scheme adds a distribution network area controller for information fusion of distribution network automation and digital substation automation, to make the information collection more direct and the fault elimination more intelligent when dealing with the defects and faults of the distribution network. [4-6]
The distribution network part in a substation automation system only considers the outlet circuit breaker of the $10 \mathrm{kV}$ feeder. For the fault of the distribution network, the whole feeder can only be removed by the substation, and the internal fault of the distribution network cannot be partially isolated, resulting in a complete power failure. The distribution automation system and intelligent substation system fusion scheme adopted in this paper is to take the distribution automation system as a subsystem of the substation automation system, as shown in Figure 1. The distribution automation system operates in the traditional mode and the distribution network is divided into several regions. Each region can be a feeder or a looped network, configured with a distribution network area controller. Intelligent terminal devices (FTU, DTU, TTU, etc.) in the region gather the collected information to the regional controller. [7.8] The regional controller is responsible for the collection and transmission of terminal information in the region, and also has some functions of sub-stations of the distribution automation system.

\section{Fusion strategy}

\subsection{Scheme introduction}

\footnotetext{
* Corresponding author: qianbifu@ 163.com
} 


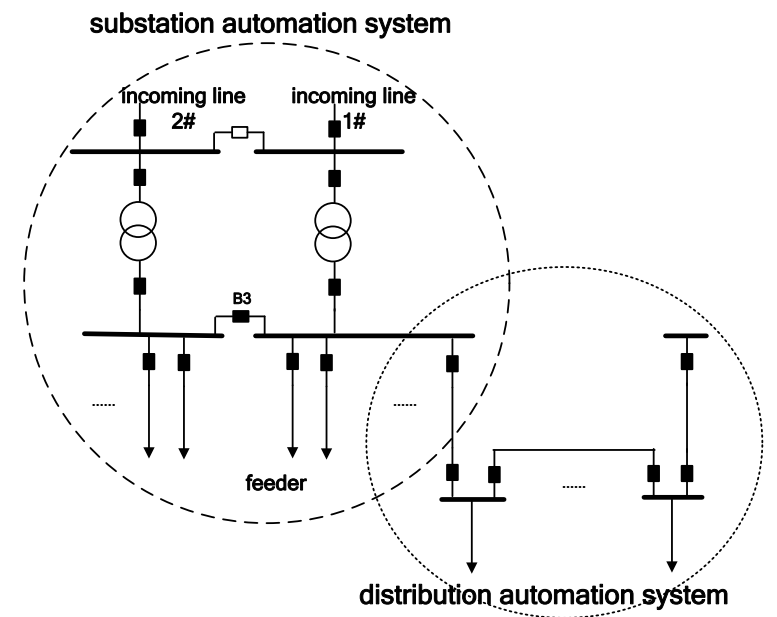

Figure 1. Schematic diagram of relationship between substation automation system and distribution automation system

\subsection{Distribution network area controller}

The traditional substation automation system and the feeder automation system are two independent systems of different levels. When the distribution feeder fails, the substation can only disconnect the fault outlet circuit breaker of the substation and make the whole outlet line out of power. While the feeder automation can only complete the fault location, isolation and recovery below the outlet circuit breaker of the substation, and has no operation right to the outlet circuit breaker of the substation. The fusion design of this paper is to add the distribution network area controller in the digital substation automation system and get through the two independent systems. [9.10]

As the central management equipment of regional distribution network, the distribution network area controller can be installed in the substation, and access the digital substation system according to the standard of IEC 61850. The substation can indirectly manage the operation and control of the distribution network equipment through the distribution network area controller. The distribution network area controller is responsible for the information interaction between the digital substation automation system and the distribution automation system. In this way, it is not necessary to connect all the equipment in the distribution network system according to the IEC61850 standard. [11] For substations, as long as the IEC 61850 model of the distribution network area controller is carried out, making the distribution network area controller conformed to IEC 61850 standard, thus the controller can be included into the substation automation system, as shown in Figure 2.

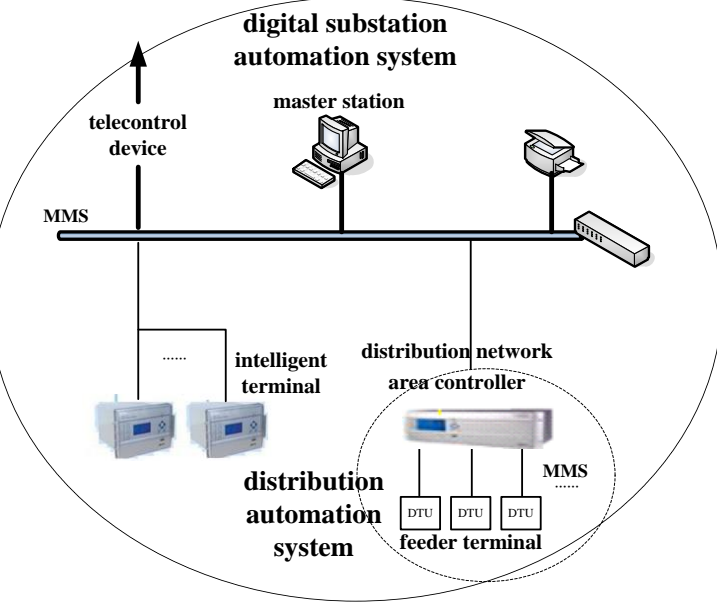

Figure 2. Digital substation network system diagram

The main contribution of this controller to the distribution network area is: when the distribution network fails and the $10 \mathrm{kV}$ feeder circuit breaker is opened, the entire intelligent system can automatically perform fault troubleshooting, locating and isolating in the distribution network area, and then reclose the feeder circuit breaker to recover power supply. Compared with the current conventional solution, which is reclosing or manual elimination, the information fusion strategy can greatly shorten the power outage time of distribution network and ensure the safe and stable operation of the power grid.

\section{Case study}

This paper aims at the distribution lines composed of one switching station named Huichang and four switching rooms named Changzutuan, Xishanqiao, Shengbei and Jinzutuan, all belonging to $110 \mathrm{kV}$ Xueshan Substation. Each switching station and switching room is configured by one DTU, and the substation is configured by a distribution network area controller. All the devices are installed in the substation, and the protection action information and the circuit breaker position information are obtained from the protection and monitoring devices of the circuit breakers B1 and B2 through a tele-control device. The network structure is shown in Figure 3.

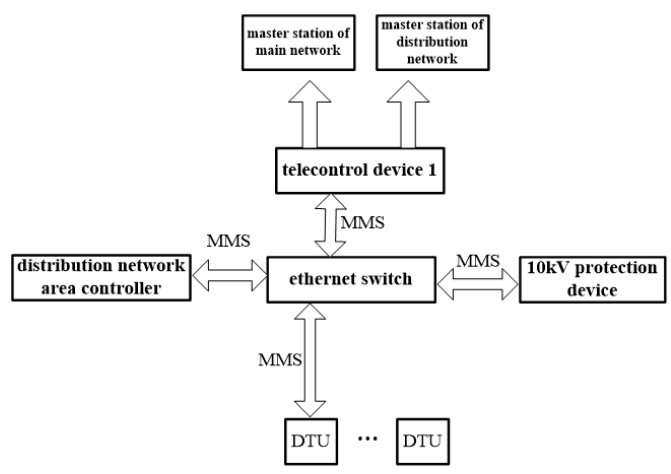

Figure 3. Network structure diagram after information fusion

The distribution network area control scheme realizes the control and feedback function of the DTU in the 
switching station and the distribution network area controller in the substation through GOOSE and SV. In addition, the definition, delivery, and control of the fault handling plan are implemented by the inter-station IEC 61850 protocol, and the message format is the MMS message. In order to achieve effective control of the master station and each terminal, different subscription and sub-area control technologies are adopted to realize fast information transmission and control at different levels and in different areas, as shown in Figure 4.

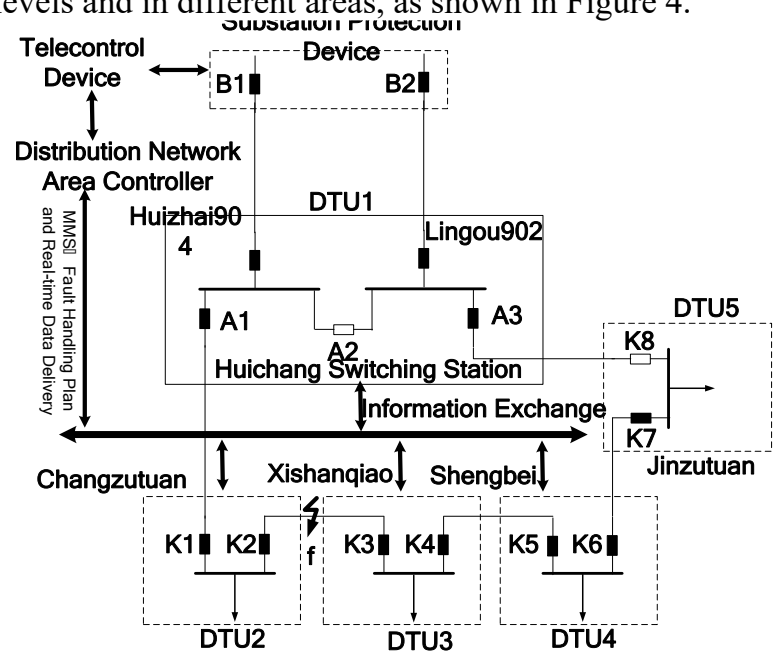

Figure 4. Network diagram of the instance

First, for different levels of distribution network terminal DTUs, the information is filtered at the first level through the VLAN domain division with priority, that is, each DTU is delimited to a VLAN, to ensure the validity and security of data transmission. In order to further ensure the information transmission organization, the second level of information is organized in the domain and the DTU in the switching station sends the circuit breaker position and current data to the distribution network area controller. The circuit breakers in the same switching station have no information to meet, which can reduce unnecessary information processing between terminals and ensure the reliability of information transmission between terminals.

On the other hand, terminals are further divided by subscription, so as to achieve the purpose of information classification and processing and improve the efficiency of information processing. As a real-time application message, GOOSE mainly transmits real-time circuit breaker position signals, protection blocking and trip signals, while SV mainly transmits current signals of corresponding nodes in the distribution network. The information carrying capacity of both is weak, which cannot meet the requirements of troubleshooting plan transmission. In order to achieve the effective transmission of the plan, the distribution network area control scheme uses the inter-station IEC 61850 messages mapped to the MMS to complete the delivery and control of the plan, that is, the DTUs and the distribution network area controller exchange MMS messages.

The current fault process of $10 \mathrm{kV}$ feeder circuit breaker in digital substation is no longer repeated in the article. The process flow after information fusion is introduced as follows. First, as shown in Figure 4, after the failure of the distribution network at point $\mathrm{f}$, the B1 breaker trips, and at the same time, the distribution network area controller receives the fault command and enters the analysis and processing mode. Second, each distribution network terminal DTU sends fault information to the distribution network area controller, and the controller performs fault diagnosis and fault location according to the distribution network control strategy. Since the fault current is detected at the circuit breaker K2, with the current direction directed to the fault point, and the circuit breaker K3 side has no fault current, it can be determined that the fault point is located between the two circuit breakers. Third, the isolation measure to disconnect $\mathrm{K} 2, \mathrm{~K} 3$, and close $\mathrm{K} 8$ is selected and delivered to the corresponding distribution network terminal DTUs (DTU2, DTU3 and DTU5) through IEC 61850. Fourth, after receiving the completion notice sent by the master station, each terminal enables the corresponding calling service, matches the corresponding troubleshooting plan automatically according to its own operating condition, performs the accident handling, and then sends a completion signal to the distribution network area controller. Finally, the controller reports to the substation automation system that the fault isolation has been completed and the power supply can be restored, thus the $10 \mathrm{kV}$ feeder circuit breaker $\mathrm{B} 1$ reclosing to recover power supply.

From the analysis of the fault processing time, taking this $10 \mathrm{kV}$ feeder fault as an example, the current effective measure is that the circuit breaker B1 directly performs a reclosing. Supposing that the sum of the protection export time and the B1 circuit breaker opening time is approximately $100 \mathrm{~ms}$, the protection reclosing time is $1 \mathrm{~s}$, and the $\mathrm{B} 1$ circuit breaker closing time is approximately $100 \mathrm{~ms}$, so the conventional total time is approximately $1200 \mathrm{~ms}$. The follow-up time of secondary trip, defect feedback (responsible department: supervision center), elimination organization (responsible department: power distribution work area), power restoration (responsible department: power transformation work area) cannot be counted due to labor, environment and other factors. However, after implementing the information fusion strategy in this paper, it is assumed that the time taken for protection export and the B1 circuit breaker opening is still about $100 \mathrm{~ms}$, the time taken for fault analysis and information interaction is roughly $80 \mathrm{~ms}$ because these are highefficiency processor behavior, the time taken for fault isolation (disconnect $\mathrm{K} 2, \mathrm{~K} 3$ and close $\mathrm{K} 8$ ) is roughly $300 \mathrm{~ms}$, and the time taken for the B1 circuit breaker reclosing is $100 \mathrm{~ms}$, so the total time is approximately $580 \mathrm{~ms}$. The efficiency of recovering power supply is greatly improved, and the network equipment is prevented from secondary impact due to reclosing at permanent fault.

In this way, through the information fusion of distribution network automation and digital substation automation, the elimination of a distribution network fault is completed accurately and rapidly. The fusion scheme proves that it can be promoted and used. 


\section{Conclusion}

In view of the fact that the current foundation of distribution network automation is still weak, it is still a long way to go to fully connect the distribution network automation system to the digital substation automation system and put it into operation in accordance with the IEC 61850 standard. Therefore, this paper adopts a scheme of fusing the distribution network automation system with the digital substation automation system. The scheme adds a distribution network area controller as an interactive bridge between the two systems, which can improve the effective utilization of station data, enhance the ability of the distribution network to handle faults, promote the popularization and implementation of IEC 61850 standard, and meet the standardization requirements of the distribution network. The feasibility of the fusion strategy is illustrated by a case study.

\section{References}

1. N. Liu, X. H. Yu, J. H. Zhang. Coordinated Cyberattack: Inference and Thinking of Incident on Ukrainian Power Grid[J]. Automation of Electric Power Systems, 2016, 40(6), pp, 144-147.

2. Q. L. Zhang, X. P. Wang, Y. Zhao. Analysis on Cascading Failures of Substation Automation System Based on IEC61850[J]. Automation of Electric Power Systems, 2013, 37(2), pp, 61-66.

3. H. S. Jiang, F. Liang, X. Yu. Digitized Substation Integrated Protection Information Sharing Communication Characteristics Analysis Research $[\mathrm{J}]$. Journal of Northeast Electric Power University, 2014, 34(2), pp, 66-70.

4. L. J. Qin, L. Liu, J. F. Shi, et al. Distribution Automation System Under IEC61850 System[J]. Electrical Equipment, 2007, 8(12), pp, 13-15.

5. L. Yuan, H. Teng. The Research of FTU Based on IEC61850[J]. Power System Protection and Control, 2011, 39(12), pp, 126-129.

6. GAO Xiang, ZHANG Pei-Chao. Main Features and Key Technologies of Digital Substation[J]. Power System Technology, 2006, 30(23), pp, 67-71.

7. W. Zhuang, L. H. Mu. Study on Cyber-Physical Relaying Protection System of Intelligent Distribution Network[J]. Power System Protection and Control, 2012, 40(4), pp, 113-118.

8. N. Cao, G. Li, D. Q. Wang. Key Technologies and Construction of Intelligent Substation [J]. Power System Protection and Control, 2011, 39(5), pp, 6368.

9. J. J. Zhang, C. X. Guo, Y. J. Cao, et al. Design of Embedded Platform for Substation Data Integration Based on Multi-Agent System[J]. Automation of Electric Power Systems, 2008,32(9), pp, 52-56.

10. S. T. Fu. Proposed Development of Smart Grid in China[J]. Automation of Electric Power Systems, 2009, 33(20), pp, 23-26.
11. B. Y. Xu, T. Y. Li, Y. D. Xue. Smart Distribution Grid and Distribution Automation[J]. Automation of Electric Power Systems, 2009, 33(17), pp, 38-41. 